\section{BASELINE GLYCOLYTIC TUMOR BURDEN PREDICTS RESPONSE AND SURVIVAL IN NSCLC AND MELANOMA PATIENTS TREATED WITH IMMUNE CHECKPOINT INHIBITORS}

${ }^{1}$ Saulo Silva*, ${ }^{1}$ Carlos Wagner Wanderley, ${ }^{2}$ Jose Flavio Marin, ${ }^{2}$ Mariana De Macedo, ${ }^{2}$ Ellen Nascimento, ${ }^{2}$ Fernanda Antonacio, ${ }^{1}$ Fernando Cunha, ${ }^{2}$ Gilberto de Castro. ${ }^{1}$ Faculdade de Medicina de Ribeirão Preto - Universidade de São Paulo, Ribeirao Preto, Brazil; ${ }^{2}$ Hospital Sírio-Libanês, São Paulo, Brazil

Background Tumor metabolic remodeling is considered one of the hallmarks of cancers and has been implicated in immune evasion. Highly glycolytic tumors can lead to immune suppression by both nutrient competition and toxic metabolite accumulation. Immune checkpoint inhibitors (ICIs) are essential in treating melanoma and non-small cell lung cancer (NSCLC) patients, but novel predictive biomarkers can significantly contribute to clinical practice. Herein we analyzed the predictive and prognostic value of baseline metabolic tumor volumes in NSCLC and melanoma patients treated with ICIs.

Methods This retrospective, single-center study includes patients with metastatic NSCLC or melanoma and a baseline 18F-FDG-PET/CT (PET) performed before ICI. PET studied parameters were SUV (maximum standardized uptake value), wMTV (whole metabolic tumor value), and wTLG (whole total lesion glycolysis). Best response rates were analyzed through RECIST. High or low glycolytic tumor burden (GTB) patients were defined according to SUV, wMTV, and wTLG cut-offs that best discriminate progressive disease. Overall survival (OS) and progression-free survival (PFS) were estimated using the Kaplan-Meier method, and curves were compared with log-rank.

Results Among 151 patients included, 107 had NSCLC and 44 melanoma. Regarding NSCLC patients, there was a significant correlation between GTB and responses among all PET parameters (figure 1). Noteworthily, only one patient with a low wTLG $(<146)$ showed progressive disease after ICIs (figure $2 \mathrm{C}$ ). In regard to the potential in predicting response to ICIs, the area under the curve (AUC) obtained for each glycolytic parameter was higher than that for PD-L1 expression (figure 2D-H). Furthermore, when patients were categorized according to ICI exposure (1st or $\geq 2$ nd line), AUC for glycolytic parameters was higher in $\geq 2$ nd line versus 1 st line (figure 3). Lastly, a low GTB was associated with improved PFS (figure 4) and OS (figure 5).Regarding melanoma patients, wMTV and wTLG were significantly associated with response (figure 6), and a low GTB was significantly associated with improved PFS and OS (figure 7).

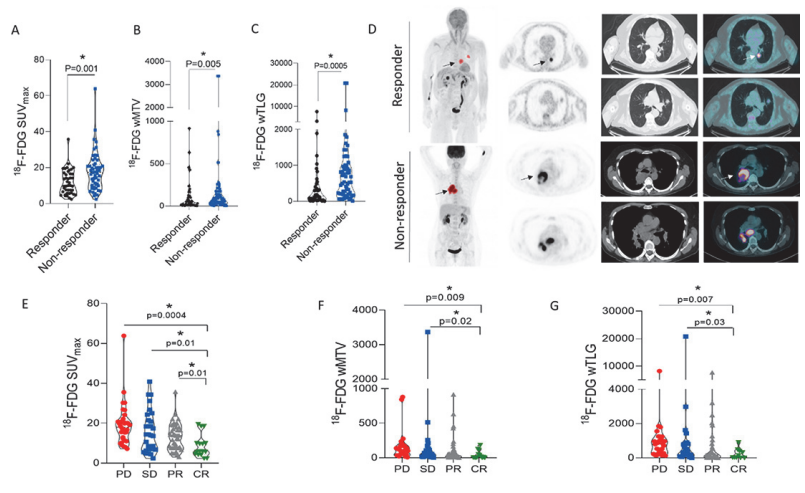

Abstract 55 Figure 1
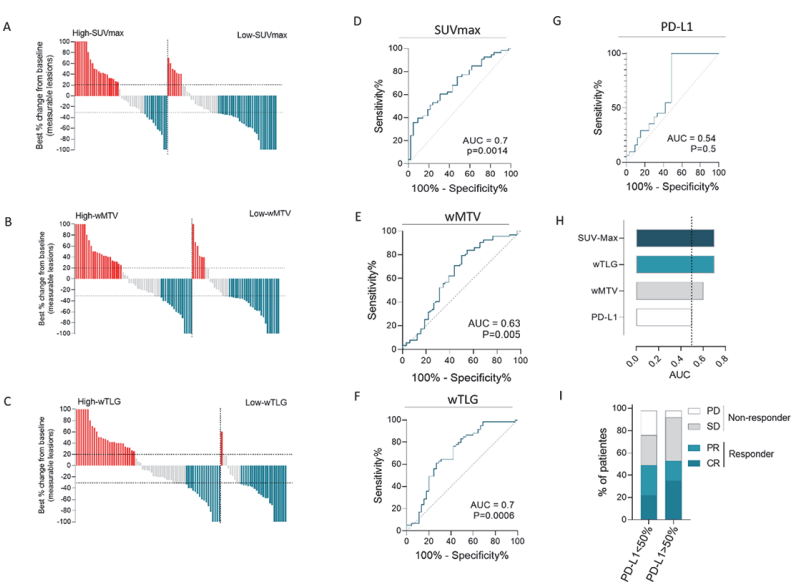

Abstract 55 Figure 2
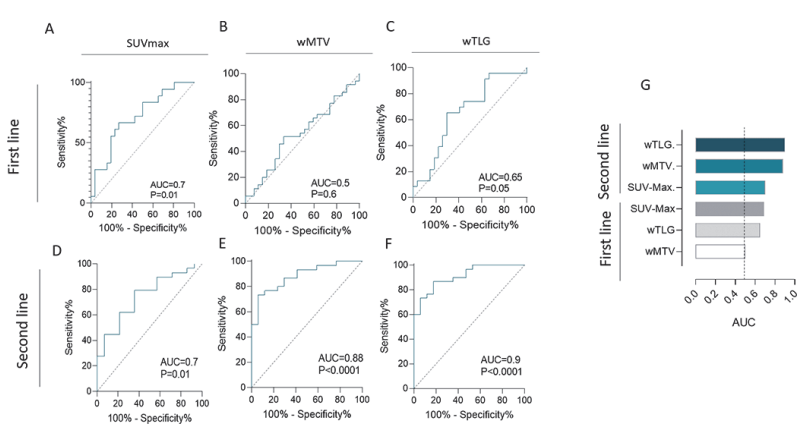

Abstract 55 Figure 3
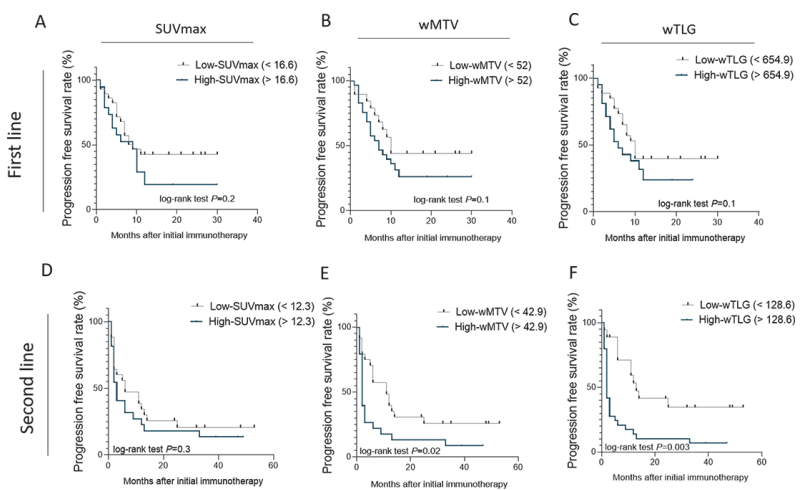

Abstract 55 Figure 4
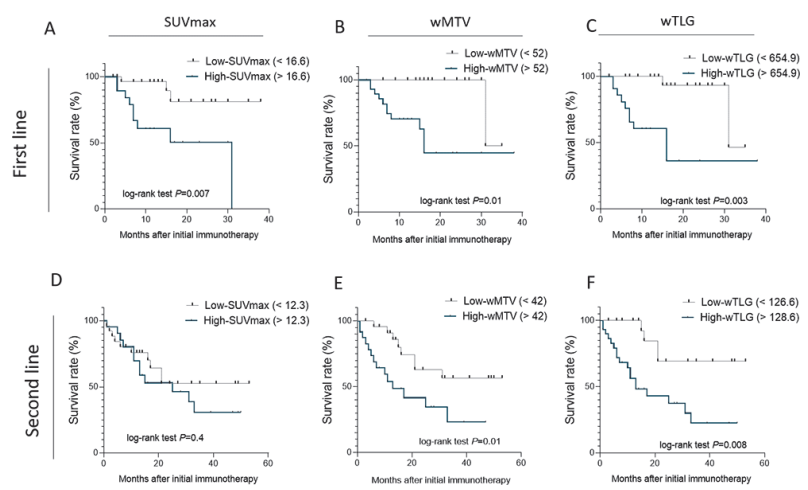

Abstract 55 Figure 5 


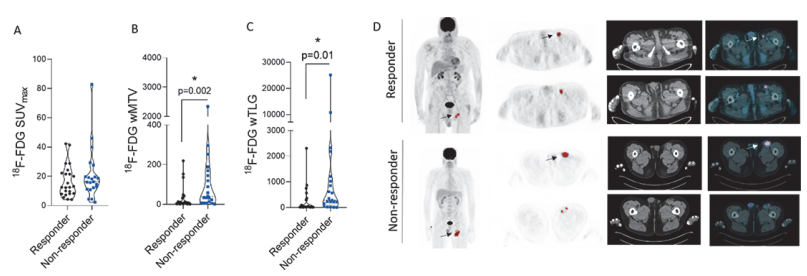

\section{Abstract 55 Figure 6}
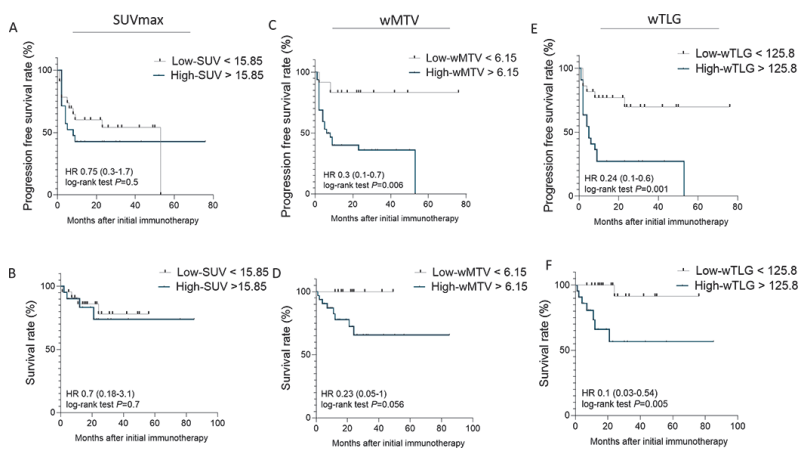

Abstract 55 Figure 7

Conclusions GTB accessed through a baseline PET is associated with survival and response to ICIs in NSCLC and melanoma patients, suggesting that the tumor metabolism might impact immune responses and be a promising predictive tool. Ethics Approval The study obtained ethics approval by SírioLibanês Hospital institutional review board. Study participants were not required to give an informed consent before taking part.

http://dx.doi.org/10.1136/jitc-2021-SITC2021.055 